PART V

THE CHROMOSPHERE IN ACTIVE REGIONS 


\title{
THE MAgNeTIC STRUCTURE OF PLAGES
}

\author{
H. ZIRIN
}

Big Bear Solar Observatory, Hale Observatories, Carnegie Institution of Washington, California Institute of Technology, Pasadena, Calif., U.S.A.

\section{Introduction}

Every sunspot group is surrounded by regions of weaker magnetic field; traditionally those parts which appear in emission in resonance lines are called plages. There is a more extensive region of enhanced magnetic field which is not bright because the magnetic field is horizontal; this region is easily discernible by dark horizontal fibrils in $\mathrm{H} \alpha$ and is included in our discussion. The plage includes most of the magnetic field in an active center and is the locus of most of the flares.

It is unfortunate that astronomers long viewed plages as westerners viewed orientals (or vice versa) as a number of indistinguishable individuals. Closer acquaintance shows that they have many distinguishing characteristics reflecting their history and magnetic structure.

For example, the spectroscopic data obtained so far on plages is hampered by the fact that there is no way of telling what part of the plage it refers to. In every case it is necessary to understand the kind of plage under discussion - whether old or new, $f$ or $p$, closed or open. It is essential to remember that the plage structure is determined uniquely by the magnetic field structure and whether it is growing or stable.

For these reasons I shall place most emphasis on the evolution of plages and their structure as determined by magnetic fields. Since this structure depends strongly on the initial evolution, we shall discuss the latter in detail. Fortunately, although plages are most varied in nature, certain basic physical and developmental patterns constantly recur.

\section{Development of Plages}

Plages are formed in the inner parts of flux loops emerging from below. Figure 1 shows such an example, a bright plage crossed by arched filament systems. These were noticed by Waldmeier.(1937) and their significance was pointed out by Bruzek (1967), who called them Arch Filament Systems. Zirin (1972) proposed the term EFR (Emerging Flux Region). In the early stages of active region growth the appearance of the group is symmetric, while a few days later the $f$ spot may disappear, leaving an extensive plage. This gives rise to the most common type of spot group, $\alpha$, with a dominant $p$ spot and a cloud of following plage, separated by a series of dark fibrils called field transition arches (Prata, 1971). The sunspots invariably form at the outer ends of the flux loops and the plages on the inside. The formation of a spot inhibits plage brightnees, probably because of the extensive horizontal field area immediately outside the penumbra, often called the superpenumbra. The formation of big $p$ spots often pro- 

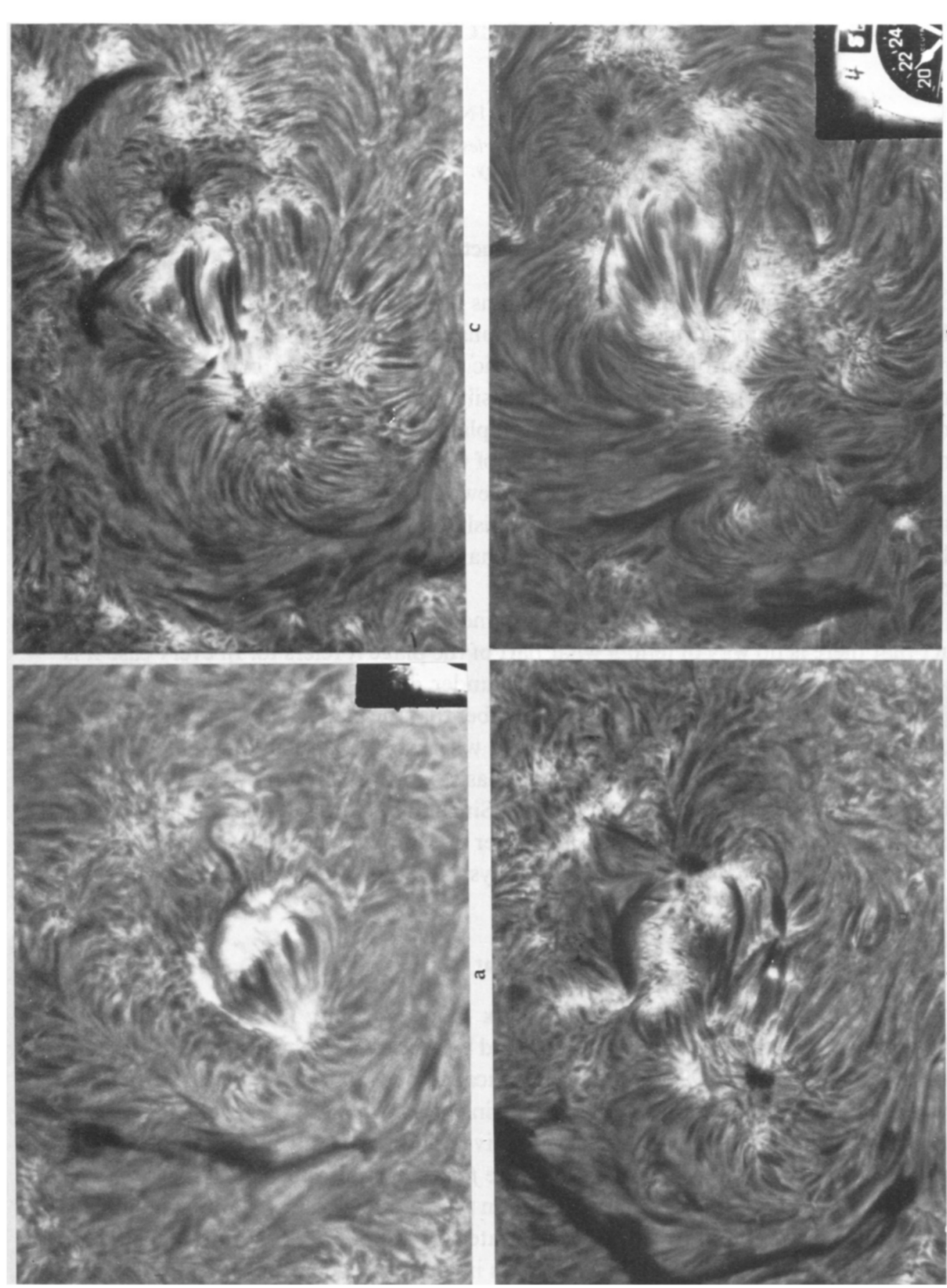

\%ั

촌

Z

E.

त्रें

뭄

吾包

可

요 3

궁

氜

- ฉ

政交

遇

है

ส

웅

인

\& 8

$\rightarrow$ 五

士 운

ป 3

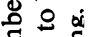

है

党

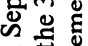

$\hat{\Sigma}:$

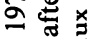

त

ชึ

$>5$

悹

절을

要

ธี

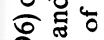

守

壳焉

ค

ए

음 웜

진

不

क्ष

皇

s.

政

즐

需焉

릉

正

定

王

- 宁

贯范 
duces a dramatic decrease in plage area, as the finely mottled plage region is replaced by horizontal field from the spot. There is also a sharp decrease in plage brightness as the field stops erupting. Further, the emerging plage is amorphous, while the mature plage is made up of fine granules. When sunspots break up a normal plage remains, so we may assume the total vertical field in umbra plus penumbra equals that in the plage. EFR's often occur in existing active regions or in close proximity to another. Since the number of EFR's per day is about two, the probability of two occurring close together is very small, and we must assume there is some connection between two or more EFR's in close proximity to each other.

The overall structure of an active region is determined both by the structure of the emerging flux loops and the sign and strength of the fields already present on the surface when it erupts. It is obvious that there must be some interaction between the emerging flux and the already existing field; although the erupting flux is closely tied together, it rapidly reconnects to nearby fields as may be judged from the fact that fibrils marking lines of force from most active regions go out to heighboring active regions. There is no evidence that existing surface flux is crowded out of the way by rising flux for the sizes of network cells on the edges of active regions are similar to those in the quiet areas.

No one has yet studied the question of the relative development of EFR's coming up in $p$ or $f$ polarity. It appears that emerging flux which is tilted to the rotation axis is more likely to produce high activity, but the smaller inclined EFR's were found by Weart to lie out earlier. If the EFR comes up in $p$ polarity, it rapidly develops an open penumbral structure, with lines of force spreading out parallel to those already present to connect with distant $f$ polarity. On the other hand, if the region emerges in $f$ polarity, a boundary filament rapidly appears to separate the new $p$ polarity from the old $f$ polarity. It is interesting that ordinary connection with fibrils normal to the neutral line never occurs, but that the boundary is normally a filament channel, with lines of force parallel to the boundary. In some cases such a boundary may be very close to the $p$ spot, even crossing the penumbra, and many flares occur at that point, but this only happens when the EFR emerges in a region of strong $f$ polarity, i.e. part of an existing spot group.

As we noted above, flux loops always emerge in simple form; within the limits of resolution, no one has ever seen a bent or twisted arch filament; the lines of force appear to go cleanly and simply from one pole to the other. The material in the loops rises at the top of the arch and drains down at the edges. It takes about $7 \mathrm{~min}$ for a $15000 \mathrm{~km}$ arch to emerge. The complexity of active regions arises from the mutual interaction of new flux loops rising within and near old ones. As the sunspots separate, a region of emerging flux loops at the center of the active region may remain. There is a strong tendency for new flux to emerge in or near existing active regions; this is in fact the source of most flare activity. Therefore, the flux tubes in big emerging regions must have a large scale complexity in the sense that each has several loops or kinks, or is accompanied by other tubes. Once formed, the arched filaments are uniform and the direction changes only if new spots or flux loops emerge. If the flux loops were 
twisted ropes, we would see complex changes in directions as they emerged, and we do not.

I should point out that there appears to be no connection between either emerging flux regions or plages and the cells of the chromospheric network. Bumba and Howard (1965) found, on the basis of low resolution spectroheliograms, that EFR's started out as elements of the chromospheric network. Weart, on the basis of superficial analysis of limited data, decided that the AFS crossed over a network cell, i.e. just the opposite. Our data shows no connection whatever between EFR's and the elements of the network. A good example is the birth of the famous August 1972 region. A recent extensive study by Harvey and Martin (to be published) finds the same result. This is a very difficult determination, because one needs extensive observation before the emergence of the new flux. Once any flux emerges, bright areas appear which are ipso facto part of the network. Logic tells us that, since the EFR's are bipolar, at most one element could be part of the local network, which must be unipolar. Hence we cannot expect new flux to be produced from manipulation of the network itself, and in fact this is not observed. Sunspots emerge from below. Nor does the network cell size have any particular relation to the sizes of EFR's, sunspots, or active regions. Since the EFR starts from zero, young EFR's are small compared to the network; mature EFR's are about $\frac{2}{3}$ the size of a network cell, but much larger EFR's have been seen. Although a cellular pattern is seen on the edges of active regions, the plages themselves are several times larger than the network and show no connection. Finally, it has been suggested by Leighton and others that the large unipolar regions grow by a random walk process in which bits of active regions break off and spread over the surface of the Sun. In 1969 Richstone made an unpublished study of the size of the enhanced network region around various active regions, and found that this area tended to grow so long as new flux was emerging, but rapidly shrank as new flux stopped rising. Similarly, observations of detailed structures near sunspots (e.g. Figures 3-5 show little or no outward motion of the plagettes, despite the fact that Sheeley (1969) and others have observed outward motion of small magnetic elements around some sunspots.

\section{Fine Structure of Plages}

Figure 2 shows a videomagnetogram made in 5324 by A. Michalitsanos compared with a simultaneous $\mathrm{K}$-line filtergram with $0.3 \AA$ bandpass made by the author. Figure 3 shows the corresponding $\mathrm{H} \alpha$ centerline and $-0.5 \AA$ pictures. Dark corresponds to $p$ polarity and light to $f$ polarity in the magnetogram. We see (after allowing for slight misalignments) in almost perfect correspondence between the pictures. Even the faint $\mathrm{K}$-line emission (except for hotizontal bright fibrils) corresponds to some longitudinal magnetic field, and we may expect that even weaker fields correspond to the weakest $\mathrm{K}$ emission. But we may be confident that most longitudinal magnetic fields are recorded by the videomagnetograph or by a $\mathrm{K}$ line or $\mathrm{H} \alpha$ picture.

We see in Figures 2 and 3 a mature $\alpha p$ type spot, with the $f$ field broken down to a plage. A simple set of field transition arches (1) separate $p$ and $f$ polarity, although a 

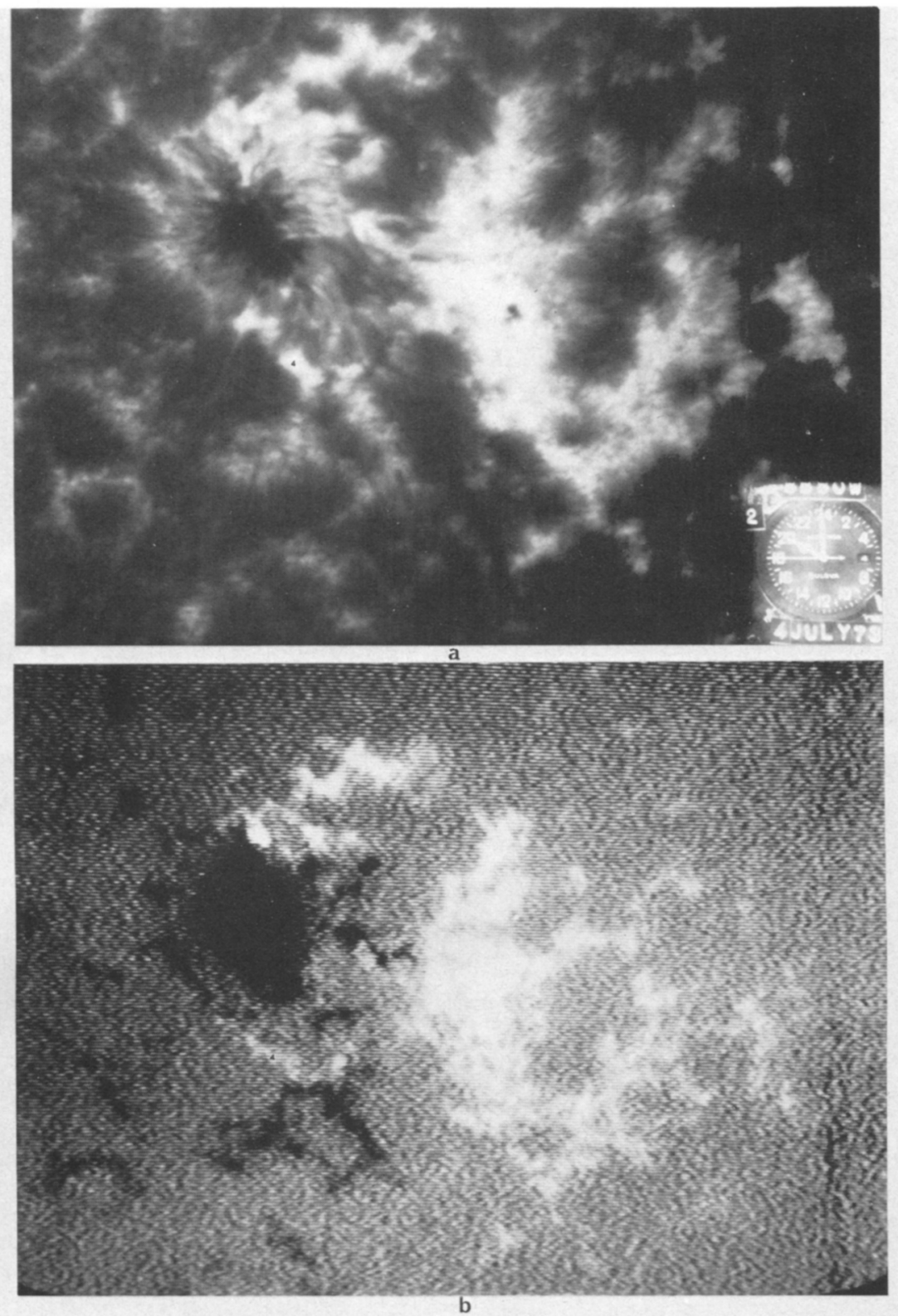

Fig. 2. (a) K line photo of McMath 12417,1973 , July 4, with $0.3 \AA$ bandpass Halle filter. This photo shows how, with good alignment and proper exclusion of internal reflections, one may obtain $\mathrm{K}$-line images comparable in quality to $H \alpha$. Careful comparison with 3 (a) shows that all bright and dark $\mathrm{K}$ features in $K$ correspond to similar features in $H \alpha$, except that the $K$ contrast is stronger and dark features in $\mathrm{K}$ are lost against the background. We have not seen running penumbral waves in $\mathrm{K}$. (b) Simultaneous videomagnetogram in 5324 made by A. Michalitsanos. The correspondence is very close. The bright rim of expanding field is marked (4). A rim of enhanced field is seen to the left of the penumbra, coinciding with a bright rim. (W is left.) 


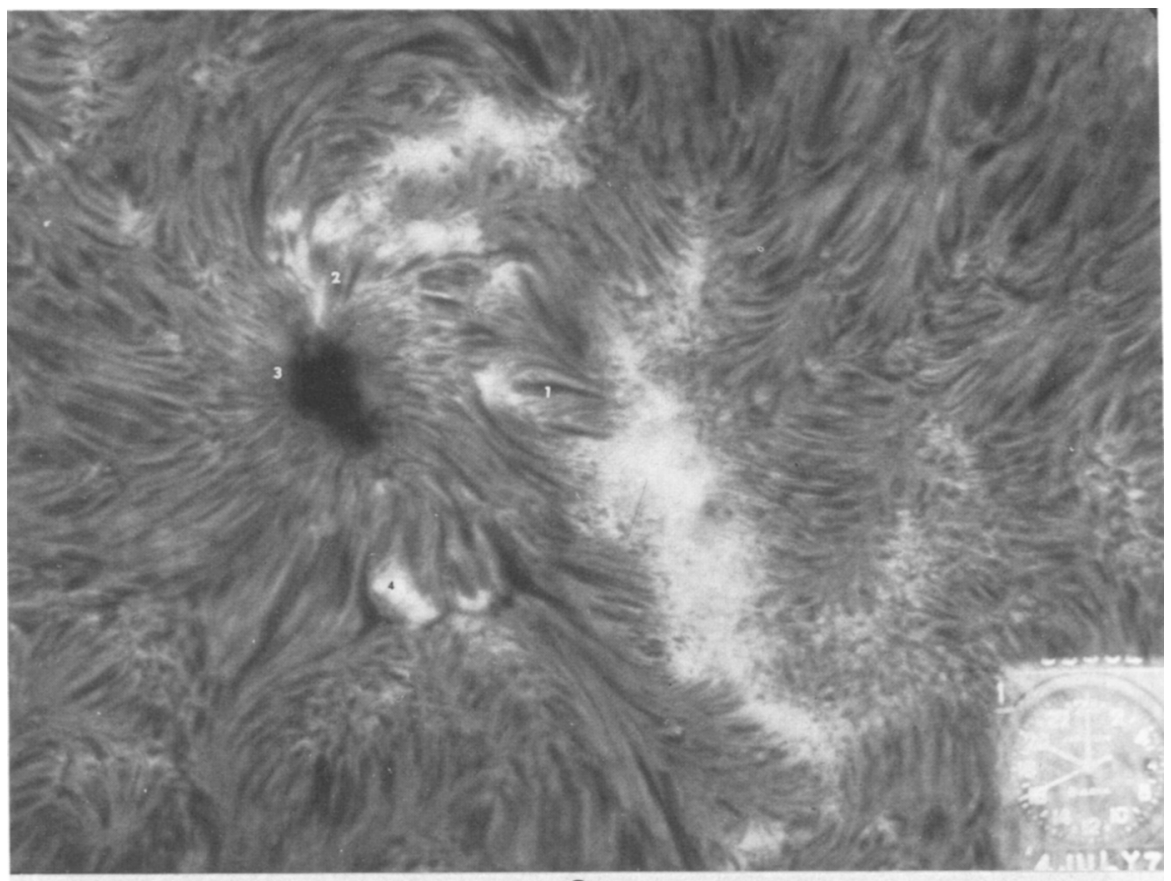

a

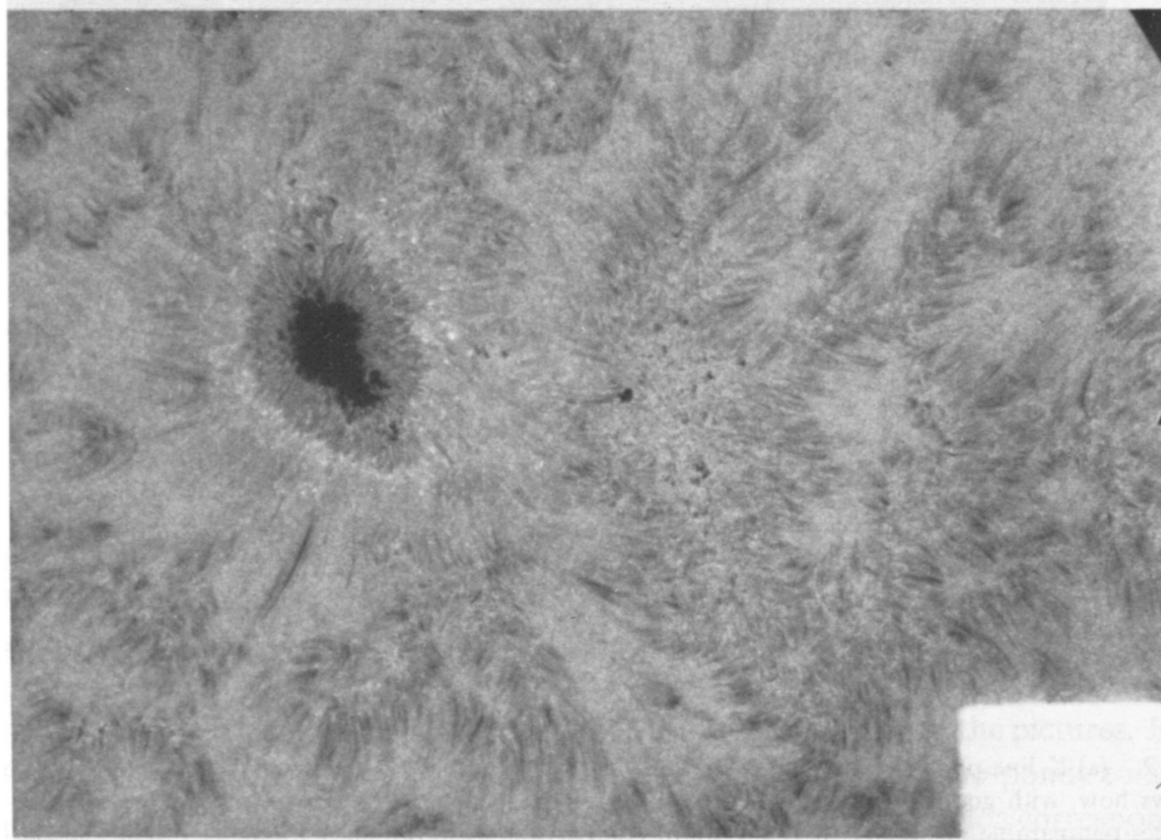

Fig. 3. (a) $\mathrm{H} \alpha$ filtergram simultaneous with $2 \mathrm{a}$ and $\mathrm{b}$. Numbers refer to features explained in the text: (1) FTA (2) more complex FTA; (3) running penumbral wave (4) expanding front of flux. (b) $H \alpha+0.6 \AA$. Note no running penumbral waves. 
more complex neutral line (2) may be seen just above the spot. The dark fibrils here are considered to follow the lines of force in the chromosphere, and indeed they invariably are found to point to the nearest opposite polarity. The magnetic structures tilt sharply away from the vertical, and, as pointed out by Foukal (1971) the stronger magnetic structures are very shallow, as shown by regions seen on the limb. One can determine magnetic field polarities and distribution by using a few simple principles:

(1) Regions of intense longitudinal fields (except spots) are bright in $K$ and $H \alpha$ centerline, but marked by fine, dark granular, structure off-band. In centerline H $\alpha$ some of these are obscured by overlying absorption, but all are seen in off-band on $\mathrm{K}$ pictures.

(2) All dark fibrils in $H \alpha$ or fine bright threads in the K-line mark the directions of lines of force; neutral lines are marked by field transition arches (systems of dark fine parallel fibrils) where the lines are perpendicular to the neutral line, and filaments (which are larger and darker) when the field lines are parallel to the neutral line. It follows as was pointed out by Foukal (1971) and by Zirin (1972), that filaments only exist when there is a shear line between opposite polarity in which lines of force run parallel to the surface for some distance and can thus support the filament and also insulate it from the corona. It is a pity that none of the present filament models confront this apparent fact.

(3) There is a rough proportionality of $H \alpha$ or $K$ brightness to field strength, except for newly emerging flux, which is brighter than normal. Jansens (1972) and Frazier (1972) have taken issue with this proportionality, but others have supported it. However, all agree that the bright $\mathrm{H} \alpha$ regions mark the locus of enhanced vertical fields.

Our data, as illustrated in Figures 2 and 3, support the correspondence of brightness to field strength, with the exception of sunspots, which are too dark, and newly emerging regions, which are too bright.

We note that the salient characteristic of the plage is a fine granular structure which may be the intersection of flux loops with the surface. This region has been shown by Tanaka and Bhatnagar to oscillate in brightness with a 5-min period. In some cases (Zirin, 1972; Figures 5 and 1, Sept. 6, 1970) these may be seen near the limb to be somewhat curved. It is remarkable that there are no spicules or fibrils coming out of uniform plages; these phenomena are only found in plagettes (the small plages of the network) or at the edges of larger plages. The effect is probably due to two factors: first, because of the strong closed magnetic fields in active regions, the flux loops from the plages turn over steeply and return to the other polarity, and second, the temperature rises much more rapidly to coronal values in the region above the plage, thus evaporating any potential spicules. The horizontal field region under the field transition region (1) appears to be heated nearly as much as the underlying plage, since it appears bright in broad band $\mathrm{K}$ pictures. The darkness in only due to the overlying $\mathrm{H} \alpha$ absorption of the field transition arches and we may conclude that horizontal field regions are also heated, but not enough to evaporate the field transition arches. 
Dunn (unpublished) has shown the existence of a fine filigree network in the upper photosphere which may possibly be the locus of the magnetic fields. The filigree is much finer than the $\mathrm{H} \alpha$ centerline structure, and this may indeed indicate that the magnetic fields spread out with height. A final determination awaits the obtaining of finer scale magnetograms.

Figures 2 and 3 show examples of other interesting phenomena. At (3), in the sunspot penumbra, (Figure 3a) we see a running penumbral wave. At (4), we see a band of $p$ polarity which moved outward from the spot over a period of days, finally annihilating when it reached the nearby $f$ polarity (Roy and Michalitsanos, submitted to Solar Physics). The magnetogram shows clearly the ragged distribution of flux. The $(f)$ flux above resulted from a small EFR there.

It is instructive to compare the appearance of active regions in $\mathrm{K}$, and various parts of $H \alpha$, because different heights are seen and different brightness contrasts. We note first that the contrast between excited regions and the background in $\mathbf{K}$ is much greater, presemably because the $\mathrm{K}$ line lies on the exponential part of the Planck curve. Although the $\mathrm{K}$ line is supposed to be formed higher, the narrowness of the $\mathrm{K}$ absorption makes certain features more transparent in $\mathrm{K}$ at the bandpass $(0.3 \AA)$. Thus we see a bright ring around the penumbra which is obscured by dark fibrils in $\mathrm{H} \alpha$ centerline but appears bright off-band.

One of the remarkable features in $\mathbf{K}$ is the network of fine bright fibrils extending over the surface, parallel to the dark $\mathrm{H} \alpha$ fibrils. At first these appear mostly bright, compared to the $\mathrm{H} \alpha$ fibrils. But careful comparison shows that this is only because the background is brighter in $\mathrm{H} \alpha$; dark features in $\mathrm{H} \alpha$ are dark in $\mathrm{K}$, and the bright $\mathrm{K}$ threads correspond to bright $\mathrm{H} \alpha$ fibrils which are barely visible onband and not visible at all off-band. Some of the $\mathrm{H} \alpha$ emission may be due to scattering of Dopplershifted photospheric $\mathrm{H} \alpha$ (Zirin, 1969) an effect which cannot give off-band emission. The evidence is that spicules are both bright and dark, and that bright and dark spicules always occur simultaneously.

An important part of the apparent bright-dark structure must be due to the illumination of spicules by the photosphere below. Since the spicules are optically deep in $\mathrm{H} \alpha$ and $\mathrm{K}$, their bottoms bask in the sunshine and are bright, while the tops are dark. These bright-dark pairings may be responsible for the doubling of spicules noted in off-band pictures by Tanaka (1973). Of course the comparison is not perfect because of quantitative contrast differences, but in principle all features correspond. Because of the contrast differences, the $\mathrm{K}$ pictures are best for determining the locus of bright features, while $H \alpha$ is best for dark fibrils and the horizontal fields. Since the resolution of these filtergrams is better than magnetograms, they are very useful for mapping the magnetic structures.

An important part of the structure of active regions is the relatively low height of the flux loops. This is demonstrated by limb pictures and also by the beautiful X-ray pictures made by AS \& E. On the other hand, the presence of higher loops is indicated by the more vertical spicule structures. The low, flat structure of active regions is a major puzzle. If it is force-free, the currents are large. 
There has been considerable interest in the possible diffusion of flux away from active regions. In general our observations do not confirm such a migration. Figure 4 shows the region on July 5 and 6 in the $K$ line and we see there has been very little change, with some small expansion to the right $(E)$ but shrinking at the top $(S)$. Certainly there is no definite pattern of expansion into unipolar regions as envisioned in Leighton's (1964) model. Yet unipolar magnetic regions must form, and flux is only separated on a sizeable scale in active regions. Although the bipolar flux in active regions emerges tightly coupled, $p$ to $f$, the region rapidly achieves a state such as we see, in which all the flux at the outer fringes connects to somewhere else. This means that a sizeable amount of flux reconnects to somewhere else, i.e. distant plage. It may be that the unipolar magnetic regions are formed, not from the elements of the active regions, but their counterparts. Thus a large active region should be followed by a unipolar region of opposite polarity. The mechanism of reconnection of flux to distant poles is unfortunately not understood.

\section{Changes in Plages and Active Regions}

As noted earlier, major changes in active regions only take place in the following ways: (1) sunspot formation and break up; (2) flux outflow from sunspots; (3) new flux emergence; and (4) magnetic field reconnection.

In general there is no proper motion at all in the plage or the surrounding plagettes except for the latter two. The formation of sunspots is of course connected with EFR's, and large complex spots may be formed by the merger of $p$ spots. When spots break up there is no particular enhancement of the plage left behind. This may be because the plage brightness is not so sensitive to the field strength for strong fields, or because the total field in the spot plus penumbra is not much greater than the surrounding plage.

We have mentioned the role of new emerging flux in producing complexity in sunspot groups. In my experience this is the principal progenitor of flares. A beautiful case is the August 1972 spot group (Zirin and Tanaka, 1973) which had completely reversed polarity from the start. The large activity in the group began when a new pair of spots began to develop and the leader, a $p$ spot, plowed into the other flux. As we mentioned above, because the lines of force in such dipoles are tightly closed, the boundary with nearby flux becomes a shear boundary storing up the energy of the spot motions for release in flares. The ultimate source of this energy is of course the magnetic buoyancy of the flux loops.

In Figure 5 we see how a new emerging flux region developed in McMath 12417 on July 8 , producing in a number of flares as it expanded, and eventually producing a second $p$ spot as the region rotated off the disk. The likelihood of this new spot group emerging at random in the center of the one sizeable spot on the disk is negligible, so we must conclude it arose from some convolution in the same flux loop. The new EFR does come up in an area where some small emerging flux was seen June 30 and July 3 , but otherwise there is no forewarning. 

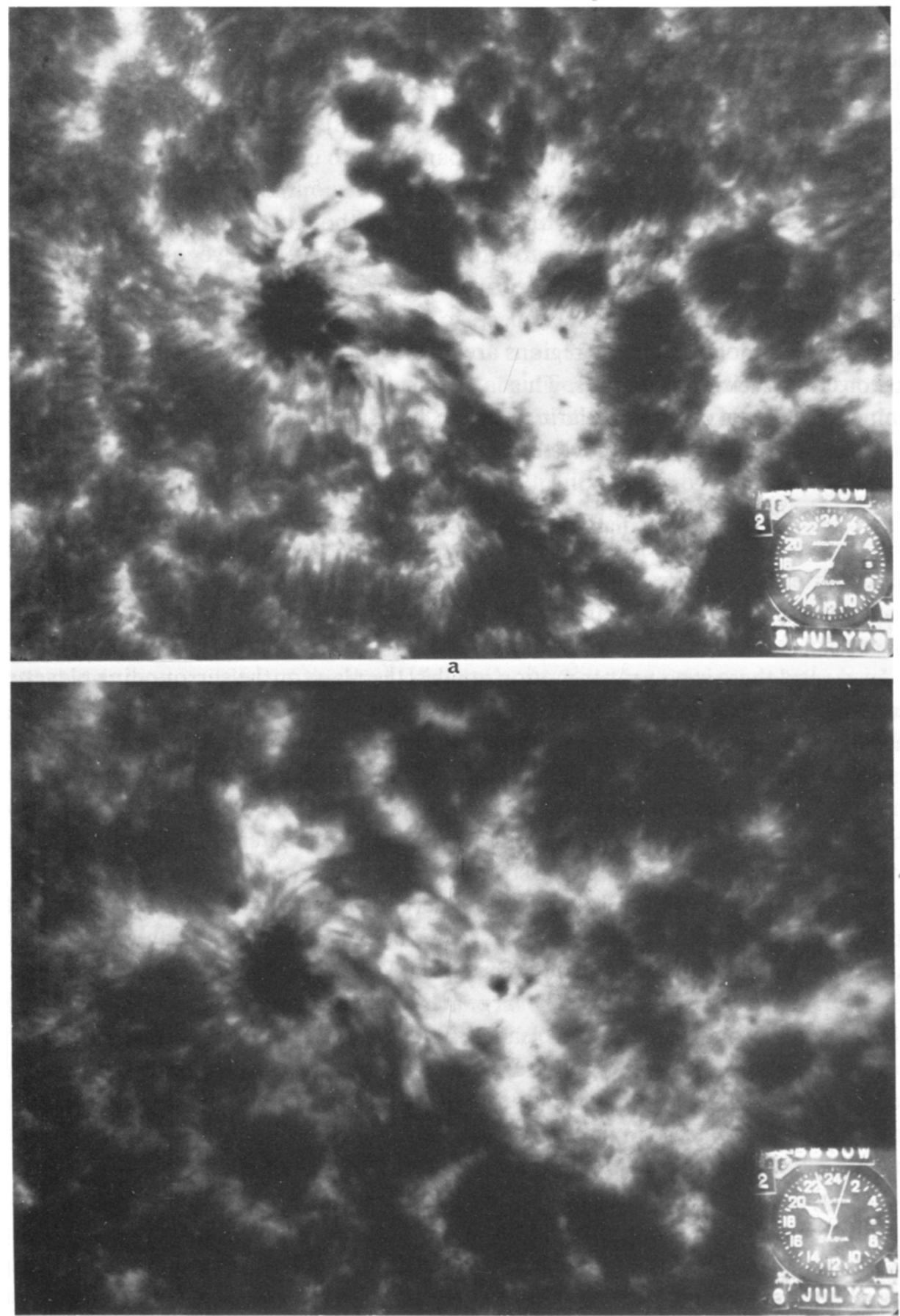

b

Fig. 4. (a) K line on July 5. (b) K line on July 6 , showing the stability of structures since July 4 (Figure $2 a$ ). Brightness changes are due to printing variation. 

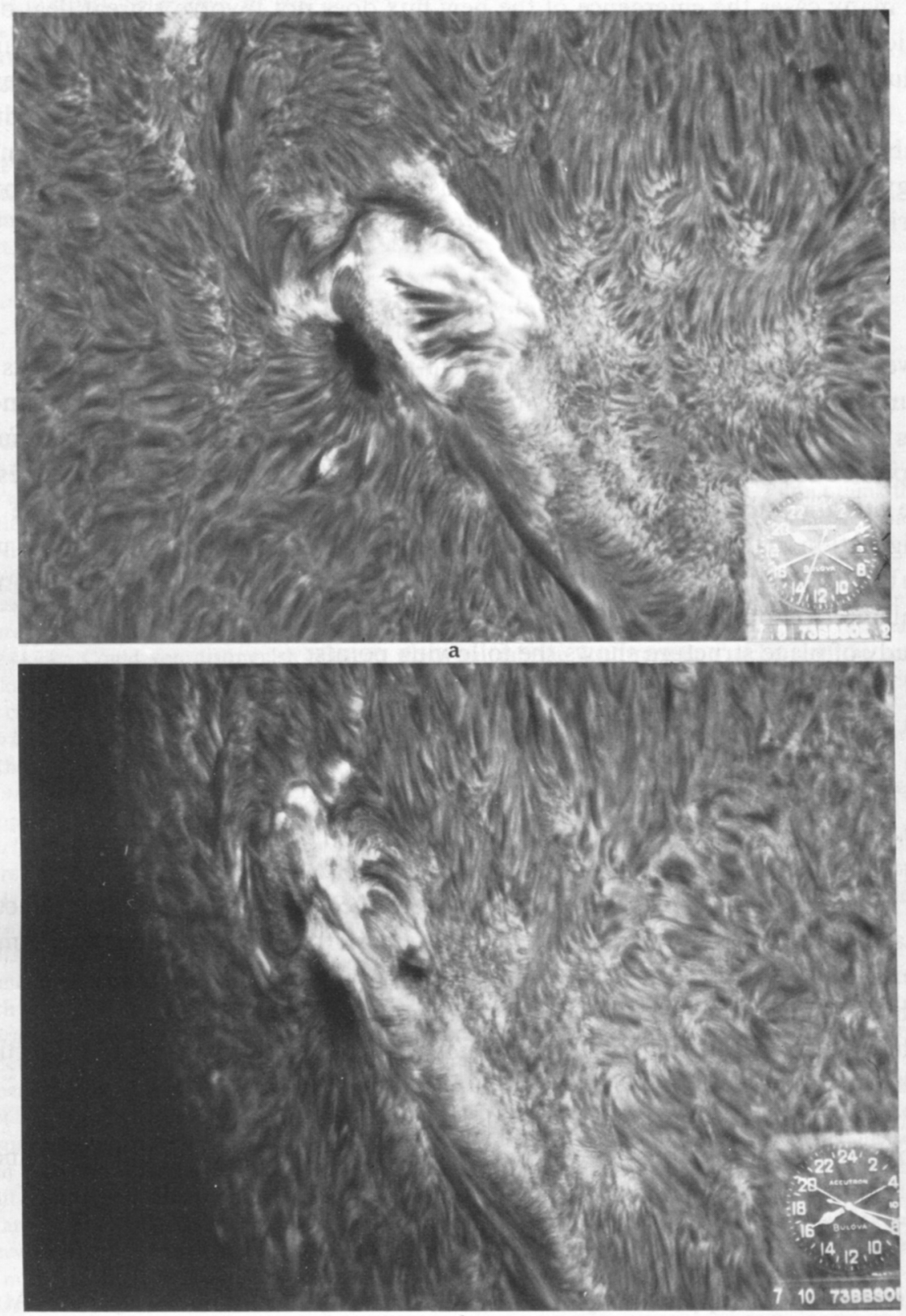

b

Fig. 5. (a) Development of an emerging flux region in McMath 12417 on 1973, July 8 . The emerging flux is marked by bright $\mathrm{H} \alpha$ emission crossed by dark arched filaments following, in this case, the previous polarity orientation. (b) Same region, July 10, showing how two new sunspots formed from the EFR. The fact that this new flux emerged exactly in the only active region on the Sun shows that flux emergence is not random but connected with or focussed by existing sunspot groups. However, EFR's outside of sunspot groups are distributed at random (Glackin, 1972). 
In many cases the emergence of the new flux does not involve a great deal of expansion, but because the flux does not arise exactly at the previous neutral line it produces a very jagged neutral line. A particularly impressive series of flares on 1972, February 15, was produced by such an effect. Field reconnection produced sizeable flares and left a smooth neutral line. The ultimate effect of reconnection and energy release is to leave an old plage consisting of two bright regions of opposite polarity connected by a fairly regular set of field transition arches.

\section{Summary}

I have tried to show that with modern high-resolution synoptic measurements and the understanding of the magnetic structure, a plage is transformed from an anonymous solar feature to a structure which reflects its history, development and future prospects. I should hope that those who carry out physical analysis of such plages pay more attention to the exact nature of the plage they are measuring and the field configuration at the point under study. Modern XUV results should enable us to learn exactly what the physical conditions are over various parts of the plage, but more visual spectroscopy is needed to define the chromospheric parameters.

Study of plage structure shows the following points:

(1) Plages emerge in the interior of emerging flux loops.

(2) Complexity is only produced by the emergence of new flux loops.

(3) There is no twist in the emerging loops.

(4) There are no spicules over plages.

(5) The plage structures are long lived.

(6) The magnetic structure of plages is quite shallow.

(7) The brightness of plages is uniquely determined by the magnetic field structure.

I have mentioned a number of important problems that remain to be solved. Among these are:

Why is a sheared magnetic boundary so characteristic and stable?

Why is the plage so much brighter when flux is emerging? (probably reconnection).

Why do plages form at the inside of flux loops, and sunspots at the outside?

Why are spicules suppressed over plages? (probably transition zone hating)

How do unipolar regions form? What is going on at the opposite end of the lines of force that we see?

\section{Acknowledgments}

This research was supported by NASA under NGR 05-002-034 and by NSF Atmospheric Sciences program under GA-24015.

\section{References}

Bruzek, A.: 1967, Solar Phys. 2, 451.

Bumba, V. and Howard, R.: 1965, Astrophys. J. 141, 1492.

Dunn, R. B.: 1972, unpublished. 
Foukal, P.: 1971, Solar Phys. 20, 298.

Frazier, E. N.: 1972, Solar Phys. 24, 98.

Glackin, David L. : 1973, Publ. Astron. Soc. Pacific 85, 241.

Janssens, T. J. : 1972, Solar Phys. 27, 1972.

Leighton, R. B.: 1964, Astrophys. J. 140, 1547.

Prata, S. W.: 1971, Solar Phys. 20, 310.

Sheeley, N. R., Jr.: 1969, Solar Phys. 9, 347.

Tanaka, Katsuo: 1973, BBSO \#0115.

Waldmeier, M.: 1937, Z. Astrophys. 14, 91.

Weart, Spencer R.: 1970, Astrophys. J. 162, 987.

Zirin, H.: 1969, Solar Phys. 7, 243.

Zirin, H. : 1972, Solar Phys. 22, 34.

Zirin, H. and Tanaka, K.: 1973, Solar Phys. 32, 173.

\section{DISCUSSION}

Beckers: I just want to make a comment about the supergranulation and the network. You intermix those two things. There is some correlation in the quiet Sun between network and the supergranulation but I am not convinced that at any point on the Sun there is such good correlation. I don't think that the correlation between brightenings in the image you have shown here and the supergranulation has been established in the active regions. I would prefer that we stick to the term network.

Zirin: I will show a slide later showing $\mathrm{CN}$ observations. I believe they are the same but so far as this discussion is concerned what I am talking about is the network as seen in $\mathrm{K}, \mathrm{H} \alpha$ or in magnetograms. This picture is a magnetic picture and defines the rise of this new region relative to the magnetic field patterns.

Newkirk: Could you distinguish between what you call the field transition arches and the emerging flux loop. Are the field transition arches simply flux loops connecting regions of opposite polarity that have ceased to emerge?

Zirin: Yes, that's right. They also have a very narrow profile and connect old flux regions that are close together.

Mme Pick: What is the relationship between the appearance of new emerging flux and the appearance of opposite polarity around the sunspots?

Zirin: I do not have a systematic answer. I think they are a different phenomenon, that is the emerging flux region is a characteristic unique thing with a life of its own whereas the parasite polarity is something that is continually either erupting or appearing from a folding downward of flux loops. I don't understand parasite fields.

Altschuler: Two questions. First, do you always find from your observations that flares are preceded by emerging flux and with what time delay, and second, can you rule out once and for all models of flares which store the initial energy in the corona? Maybe in a neutral sheet?

Zirin: There is not a direct temporal relationship. Flux emerges and this produces a distortion in which the new flux has stressed the magnetic field which must reconnect, so this produces flares, but that doesn't happen in an hour or six hours or even twenty four hours after the flux emerges. However, if flux does not erupt there are no flares except perhaps for the small flares. There are lots of little things happening around the sunspots but larger flares occur when the flux erupts. In answer to your question, my thoughts are very much turned towards the chromosphere because this is what we look at. I cannot rule out anything that might be going on in the corona. You always tend to think that what you are looking at is the most important part. I would say this in the tight active regions. I think it is all stored down below because of the observations that Tanaka and I made in the August flares where you see a highly stressed magnetic field low in the surface and then afterward you see nice potential loops.

Athay: In your discussion of emerging flux regions outside of active regions you implied that they occurred with equal probability at all solar latitudes. Did you really mean this?

Zirin: No, they occur with equal probability in longitude but in latitude they occur primarily in the sunspot zones. The butterfly diagram is the end result of the EFR's and they do occur most frequently at the same latitude that has the sunspots still occurring.

Beckers: You raised the point a few times that the emerging flux loops and the field transition arches are horizontal structures. The same thing is true of the fibrils and the vortex structure that you see around the sunspots. I have often asked why it is that way and I like to give myself the following answer. If there is a bipolar magnetic region on the Sun and nothing but that bipolar magnetic region there will be lots of 
field lines that connect the two bipolar regions. Some of these will lie in the horizontal plane and some will rise above the horizontal plane extending into the corona. The ones in the corona will be emptied of their matter and will disappear, the only ones that will remain visible in $\mathrm{H} \alpha$ are the ones that are horizontal and lie at a low level in the atmosphere. If there is a much more confused magnetic field for example, a magnetic field concentration between the dipoles the picture would be distorted but I don't know in what way. I have been wondering whether the regions between these arches and below the arches may be completely void of field in the lower levels. I would just like to throw that out as an explanation.

Zirin: I would say that explanation is by and large right. However if one looks with high resolution at these regions near the surface the field lines seem to be nearly horizontal with very little vertical extent. I agree that our observations are very biased by the fact that it would be difficult to see enough neutral hydrogen to show the arches in the corona. When a flare occurs it normally illuminates those loops, but maybe that's just because we fill those loops up with material when a fiare happens. Maybe those are real flux loops but I doubt it. However at the limb we still see these very low arches that are sharply bent over and are not potential fields they seem to require large current. Some people object to this but I have no control over it myself.

Giovanelli: I don't see any problem at all with the low arches. That is just what I would predict if there is a tight magnetic field below the photosphere; as it rises up it will expand because of the reduced gas pressure. As soon as it gets up above $1000 \mathrm{~km}$ it is surrounded by corona where the pressure is nearly constant over a considerable range of height. This means that a flux loop will become more or less uniform in diameter and the least energy is involved when the tube of force goes straight across between the two ends. According to this picture the tube of force rises up and expands until it is more or less of uniform diameter and then goes straight across to its other end.

Zirin: Someone needs to study these things, the observational material exists and the theoreticians have not really had a crack at it.

Martin: I would like to state a qualification about your statement about the number of active regions that form per day. Weart found that only two active regions form per day but this pertains only to active regions that attain the size of the supergranule cells or larger. For regions that don't attain this size and may not even form pronounced sunspots the number is very large. Karen Harvey and I found that the number is between 50 and 100 emerging flux regions per day.

Zirin: Yes, I was surprised at that big number that is on our docket to look at from our data. I agree that two per day is two active regions per day that reach supergranule size and form sunspots. We see the little ones but I don't see 50 per day.

Martin: The 50 per day was based on the count for the entire sun, and has been verified with Kitt Peak magnetograms taken since the beginning of the Skylab programs. On one magnetogram a day, which is insufficient to see all of the emerging flux regions we actually see 10 to 20 per, day so that we figure that with more complete observations of the whole Sun we will be seeing the larger number.

Zirin: I think you will agree that there are only about 2 per day that attain large size, and therefore my conclusions about the probability of erupting within a sunspot group are not affected by that statement.

Martin: I agree with you but $I$ think that the difference is that emerging flux is very common in active regions.

Newkirk: I am worried about your statement that flux emergence and field line reconnection accounts for practically all of the flux. Unless I misunderstood you this would imply that all of the flux is essentially annihilated locally in the active region. This is contrary to the picture that most of the flux in extended unipolar regions is flux that is just torn off from active regions. If you annihilate all of the flux in active regions you are then never going to get field lines connecting widely separating centers unless you can come up with some technique for preferentially destroying one field sign over the other.

Zirin: I thought I had addressed that question but maybe you missed it. I realize that we do not understand how flux loops connect to different places. It seems like filaments play an important role because they carry flux for such a large distance. There must be some reconnection which results in the connection of a new flux to some very distant flux somewhere else. But what I was trying to say is that one sees virtually no outward flow of magnetic flux in these plages. When new flux stops coming up the active regions shrink. The only thing I can guess is that when the plages come up, part of them gets connected to something farther out. Although we don't see the regions moving out each of the lines of force must connect back to the solar surface at some point in opposite polarity and that might conceivably account for the fact that we do not see flux expansion in the plages.

Schatten: I have recently come up with some ideas that might support Alfvén's old picture of Alfvén waves coming up from below. Can you rule that out on the basis of your observations? 
Zirin: I don't understand how I would observe hydromagnetic waves.

Schatten: Isn't that what you are talking about?

Zirin: I shouldn't have used the word waves. What we saw was a sunspot with a large chunk of magnetic flux appearing and moving outwards. That is not a common phenomenon. The principle way that flux comes up is in the bipolar loops.

Schmidt: I have a nasty comment. I happen to be the guy who is worried about the Lorentz forces. I do not think the chromosphere and corona are equal footing when we consider the flux distribution. This is a geometrical question and in my own, old fashioned mind, this is a question of $4000 \mathrm{~km}$ height estimated for the fibrils versus my estimate here of $400000 \mathrm{~km}$. 